\title{
Evaluating Low-frequency Pulsar Observations to Monitor Dispersion with the Giant Metrewave Radio Telescope
}

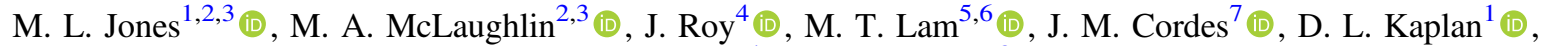 \\ B. Bhattacharyya ${ }^{4}$ (D), and L. Levin ${ }^{8}$ (D) \\ ${ }^{1}$ Center for Gravitation, Cosmology and Astrophysics, Department of Physics, University of Wisconsin-Milwaukee, P.O. Box 413, Milwaukee, WI 53201, USA \\ ${ }^{2}$ Department of Physics and Astronomy, West Virginia University, Morgantown, WV 26506, USA; megan.jones@nanograv.org \\ ${ }^{3}$ Center for Gravitational Waves and Cosmology, West Virginia University, Chestnut Ridge Research Building, Morgantown, WV 26505, USA \\ ${ }^{4}$ National Centre for Radio Astrophysics, Tata Institute of Fundamental Research, Pune 411 007, India \\ ${ }^{5}$ School of Physics and Astronomy, Rochester Institute of Technology, Rochester, NY 14623, USA \\ ${ }^{6}$ Laboratory for Multiwavelength Astrophysics, Rochester Institute of Technology, Rochester, NY 14623, USA \\ ${ }^{7}$ Cornell Center for Astrophysics and Planetary Science and Department of Astronomy, Cornell University, Ithaca, NY 14853, USA \\ 8 Jodrell Bank Centre for Astrophysics, School of Physics and Astronomy, University of Manchester, Manchester M13 9PL, UK \\ Received 2020 July 19; revised 2021 April 29; accepted 2021 May 2; published 2021 June 29
}

\begin{abstract}
The North American Nanohertz Observatory for Gravitational Waves (NANOGrav) project has the primary goal of detecting and characterizing low-frequency gravitational waves through high-precision pulsar timing. The mitigation of interstellar effects is crucial to achieve the necessary precision for gravitational wave detection. Effects like dispersion and scattering are stronger at lower observing frequencies, with the variation of these quantities over week-month timescales requiring high-cadence multifrequency observations for pulsar timing projects. In this work, we utilize the dual-frequency observing capability of the Giant Metrewave Radio Telescope (GMRT) and evaluate the potential decrease in dispersion measure (DM) uncertainties when combined with existing pulsar timing array data. We present the timing analysis for four millisecond pulsars observed with the GMRT simultaneously at 322 and $607 \mathrm{MHz}$, and compare the DM measurements with those obtained through NANOGrav observations with the Green Bank Telescope and Arecibo Observatory at $1400-2300 \mathrm{MHz}$ frequencies. Measured DM values with the GMRT and NANOGrav program show significant offsets for some pulsars, which could be caused by pulse profile evolution between the two frequency bands. In comparison to the predicted DM uncertainties when incorporating these low-frequency data into the NANOGrav data set, we find that higher-precision GMRT data is necessary to provide improved DM measurements. Through the detection and analysis of pulse profile baseline ripple in data on test pulsar B1929+10, we find that, while not important for these data, it may be relevant for other timing data sets. We discuss the possible advantages and challenges of incorporating GMRT data into NANOGrav and International Pulsar Timing Array data sets.
\end{abstract}

Unified Astronomy Thesaurus concepts: Millisecond pulsars (1062); Radio interferometers (1345)

\section{Introduction}

Gravitational waves (GWs) offer a new window through which to study the universe, with the first direct GW detection in 2016 (Abbott et al. 2016). A low-frequency (approximately nanohertz) detection in the pulsar timing array (PTA) portion of the GW spectrum would provide information about sources (e.g., the GW background due to supermassive black hole binaries, supermassive black hole mergers, among others) that are not visible to other $\mathrm{GW}$ experiments sensitive to higher frequency GWs (e.g., Sesana 2013; Lasky et al. 2016; Arzoumanian et al. 2020; Buchmuller et al. 2020; Blasi et al. 2020). In order to detect GWs using PTAs, timing models for each pulsar must first be constructed by accounting for all known effects on the pulse times of arrival (TOAs) in order to minimize the differences between the measured and modelpredicted TOAs (i.e., timing residuals). The detection of GWs using pulsars requires high-precision timing with TOA accuracy less than approximately microseconds (e.g., Demorest et al. 2013; Arzoumanian et al. 2015). Therefore all timing fluctuations, both intrinsic (e.g., binary motion) and extrinsic (e.g., interstellar plasma) to the pulsar, must be accounted for in the timing model.

There are currently three global PTA efforts focused on GW detection through pulsar timing with decade-long data sets: the
North American Nanohertz Observatory for Gravitational Waves (NANOGrav; Arzoumanian et al. 2018), the European Pulsar Timing Array (Desvignes et al. 2016), and the Parkes Pulsar Timing Array (Kerr et al. 2020). These three experiments along with the more recently formed Indian Pulsar Timing Array (Joshi et al. 2018) form the International Pulsar Timing Array (IPTA; Perera et al. 2019). IPTA data releases are comprised of data from all eight radio telescopes used by these three regional collaborations. As more pulsar observing instruments have come online, additional PTA efforts are developing (e.g., Joshi et al. 2018; Hobbs et al. 2019; Bailes et al. 2020). The inclusion of more instruments into the IPTA can aid in producing a more valuable data set by increasing the number of millisecond pulsars (MSPs), the number of TOAs, the sky coverage, and observing frequency coverage. In addition, new low-frequency $(<200 \mathrm{MHz})$ instruments provide useful supporting measurements to PTA science, such as longterm DM and pulse broadening variations of both millisecond and canonical pulsars, that can be incorporated into IPTA analyses (e.g., Bhat et al. 2018; Bansal et al. 2019; Kirsten et al. 2019; Bilous et al. 2020).

The Giant Metrewave Radio Telescope (GMRT) consists of 30 antennas, each with a $45 \mathrm{~m}$ diameter; the total collecting area of the GMRT is equivalent to a $\sim 250 \mathrm{~m}$ diameter single dish telescope. Using six feeds, the array can observe finite 
frequency bands with centers ranging from $150-1250 \mathrm{MHz}$ (Gupta et al. 2017). By splitting the array, the GMRT is capable of executing simultaneous dual-frequency observations that can be complementary to NANOGrav observations by offering coverage at lower frequencies, where frequencydependent timing fluctuations due to interstellar effects are more prominent because of the inverse dependence on observing frequency.

\subsection{Interstellar Effects}

The dominant delay induced by the interstellar medium (ISM) in timing data is due to dispersion. As the radio pulse travels through the ISM, it encounters ionized plasma along the way. The dispersion measure (DM) is the integrated column density of free electrons along the line of sight (LOS) to a pulsar

$$
\mathrm{DM}=\int_{0}^{d} n_{e}(l) d l,
$$

where $n_{e}$ is the free electron density at position $l$ along the LOS of distance $d$ to the pulsar. DM therefore can be used to infer the distance to the pulsar by assuming a free electron density model for the galaxy (e.g., Cordes \& Lazio 2002; Yao et al. 2017). The time delay due to dispersion is

$$
t=K \frac{\mathrm{DM}}{\nu^{2}}
$$

where $K=4.15 \mathrm{~ms} \mathrm{GHz}^{2} \mathrm{pc}^{-1} \mathrm{~cm}^{3}$ is the dispersion constant (Lorimer \& Kramer 2012) and $\nu$ is the observing frequency. DM can be estimated by observing at multiple frequencies or at a single frequency over a wide bandwidth and comparing the respective time delays. Due to the changing LOS, both as the pulsar moves relative to the Earth as well as dynamic processes in the ISM, DM is not constant in time and is in reality $\mathrm{DM}(t)$, requiring epoch-to-epoch monitoring and correction (e.g., Isaacman \& Rankin 1977; Jones et al. 2017).

In addition to dispersion, interstellar scattering also causes a frequency-dependent time delay, scaling as $\sim \nu^{-4}$ and therefore much more influential at lower observing frequencies. As the pulse travels through the ISM, it will be scattered due to inhomogeneities in the ISM, which cause multipath propagation. These multiple ray paths introduce a delay in the TOA. Like DM, scattering delays are also time variable. Scattering cannot be corrected as easily as DM, but can be partially corrected using high-resolution dynamic spectra due to the similar phenomenological cause behind scattering and scintillation (Levin et al. 2016). Because of the covariances between fitting for DM and scattering, some scattering effects will be absorbed by fitting for only DM. Thus, low-frequency observations can be used to disentangle the scattering contributions from the DM through the discrimination between the $\nu^{-2}$ and $\nu^{-4}$ variations.

\subsection{This Work}

NANOGrav typically observes pulsars with a strategy designed to measure DM variations at the cost of observing time (e.g., Arzoumanian et al. 2018). Most MSPs are observed on a monthly cadence with a single telescope-either the Arecibo Observatory or the Green Bank Telescope (GBT) using observations at two frequencies: a higher frequency (typically $1.4 \mathrm{GHz}$ or above) where the timing precision is often better (see Lam et al. 2018) and a lower frequency (typically $800 \mathrm{MHz}$ or below) to provide a long frequency lever arm to track DM variations. This doubles the required observing time per source beyond the minimum required to just measure the TOAs themselves. In this work we examine whether incorporating observations from a third telescope could help anchor the DM measurements to the DM curve, increase their precision, and provide a valuable boost in observing efficiency.

Through this analysis, we help evaluate low-frequency data obtained using the GMRT for ultimate inclusion into the IPTA. We present GMRT timing data for four MSPs that are also part of the NANOGrav $11 \mathrm{yr}$ data set. We investigate the potential improvement in DM precision by incorporating this GMRT data with the NANOGrav $11 \mathrm{yr}$ data as a test case. We discuss the data acquisition and observing modes at the GMRT in Section 2. We compare predicted and actual sensitivities, compare the DMs measured at high and low frequencies, and investigate possible reasons for differing DMs in Section 3. We measure a time-dependent power fluctuation known as baseline ripple seen in pulse profiles obtained with the GMRT, and make predictions for its effect on MSP timing in Section 4. We discuss the potential for producing higher-precision DMs in Section 6.

\section{Data}

Observations were done using the GMRT phased-array mode $^{9}$, in which a subset of the full array of antennas can be phased. The phased-array mode is capable of off-line coherent dedispersion and allows the array to be subdivided into two independent subarrays, each of which has its own beam, allowing for simultaneous dual-frequency observations. In order to make the DM measurements at the GMRT useful to aid PTA sensitivity, a dual-frequency coherently dedispersed observing mode was developed. Significant optimization efforts in computing, memory, and network bandwidth requirements were employed to maintain sustained real-time streaming of dual coherent voltage beams over $32 \mathrm{MHz}$ bandwidth at Nyquist resolution. These high-gain coherent beams at lower frequencies aided by the high-performance signal processing capability make the GMRT a useful instrument to follow-up PTA MSPs for monitoring ISM parameters. Earlier work reported by Kumar et al. (2013) for measuring DM variations of PTA MSPs with the GMRT used incoherently dedispersed observing mode.

Our data were taken simultaneously centered at 322 and $607 \mathrm{MHz}$ with a $32 \mathrm{MHz}$ bandwidth at each frequency. A subset of eight antennas was centered at $322 \mathrm{MHz}$ and 15 antennas centered at $607 \mathrm{MHz}$ (a maximum of $\sim 23$ antennas can be used for phased-array pulsar observations to avoid phasing inefficiency at longer baselines). The observing parameters used here are now considered part of the legacy GMRT system after the implementation of system upgrades to form the uGMRT (Gupta et al. 2017). Predicted sensitivities with the uGMRT are discussed in Section 3.

Observations occurred at 11 epochs between 2013 February 2 and 2014 October 25. A test pulsar, PSR B1929+10, was

\footnotetext{
9 These data are from the legacy GMRT system, used through 2017. While this has been largely superseded by the upgraded GMRT (uGMRT; Gupta et al. 2017) some elements of the system are still relevant, and our analysis still holds lessons for future observations. We explicitly compare our results with predictions for uGMRT analysis in Section 3.
} 


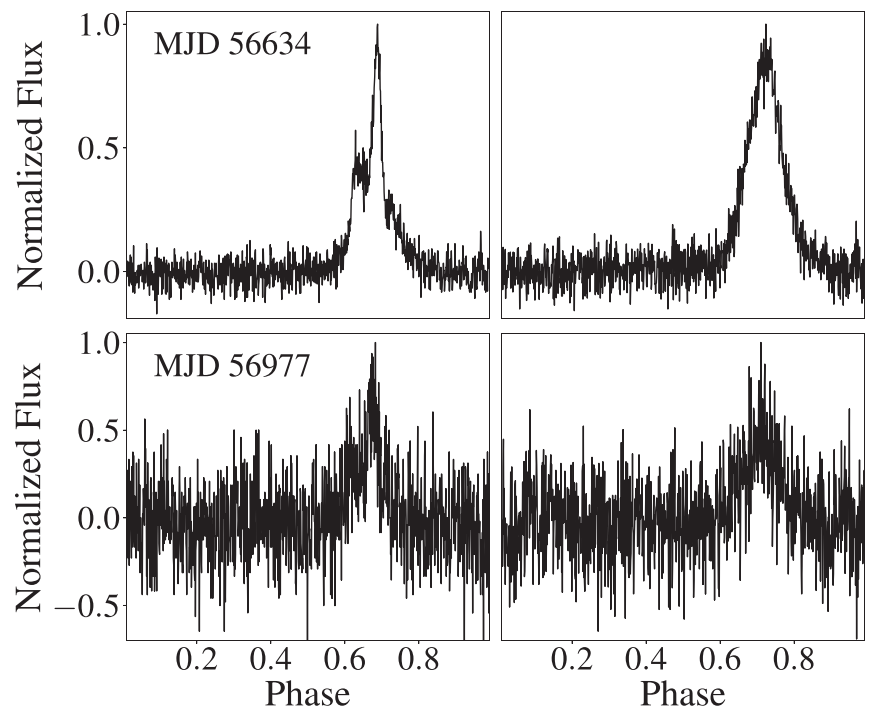

Figure 1. PSR J1640+2224 pulse profiles for two epochs of GMRT observations at $322 \mathrm{MHz}$. The coherently dedispersed data are on the left; the incoherently dedispersed data are on the right. The average TOA errors of the incoherently dedispersed data are 4 and 10 times higher (on MJDs 56634 and 56977, respectively) when compared to the coherently dedispersed data at $322 \mathrm{MHz}$. At $607 \mathrm{MHz}$, the difference is predictably less significant with the incoherently dedispersed TOA errors only double that of the coherently dedispersed data.

observed at each epoch for $\sim 5$ minutes to inspect data quality. The GMRT Software Backend simultaneously creates both coherently and incoherently dedispersed data (Roy et al. 2010); the analysis was performed on the coherently dedispersed pulsar data. DMs for coherent and incoherent dedispersion were obtained from the ATNF Pulsar Catalog (PSRCAT $^{10}$; Manchester et al. 2005). A comparison of the coherently and incoherently dedispersed data can be seen in Figure 1.

The coherently dedispersed data were split into 32 sub-bands across each of the two frequency bands, while the incoherently dedispersed data was divided into 512 frequency channels. Left and right circular polarizations were combined during processing. The data headers were inserted after the observation using a separate script as they are not encoded during the observation. Clock correction files do not exist for the GMRT and therefore were not used.

Of the 10 MSPs originally observed as part of this project, only four had a sufficient number of high signal-to-noise $(\mathrm{S} / \mathrm{N})$ detections to be used for high-precision timing. PSRs J1640 +2224 , J1713+0747, J1909-3744, and J2145-0750 were observed for $\sim 30$ minutes at each epoch. Data were folded using the DSPSR ${ }^{11}$ (van Straten \& Bailes 2010) software package. We fit multiple Gaussians for each pulsar at each frequency for the epoch producing the highest $\mathrm{S} / \mathrm{N}$ observation to produce a pulse template for calculating TOAs. Examples of pulse profiles from our GMRT observations are shown in Figure 2. PSRCHIVE ${ }^{12}$ was used for TOA generation (van Straten et al. 2012).

Fitting of the timing model was done using the TEMPO software package, ${ }^{13}$ which applies a least-squares fit to the

\footnotetext{
${ }^{10}$ http://www.atnf.csiro.au/people/pulsar/psrcat/

$11 \mathrm{http}: / /$ dspsr.sourceforge.net

12 http://psrchive.sourceforge.net

13 http://tempo.sourceforge.net
}

TOAs (Nice et al. 2015). When fitting for multiple epochs, the DM values are assumed constant for an individual epoch and encoded via the DMX parameter. TEMPO reports $1 \sigma$ errors on DMX determined from the timing-parameter covariance matrix after the least-squares timing model fit. DMX fitting was performed using ephemerides produced from the NANOGrav 11 yr data set (Arzoumanian et al. 2018). The majority of the timing parameters (e.g., positions, binary parameters) from NANOGrav are more precise than those we would obtain from fitting just the GMRT data; therefore, all parameters except DMX were held constant in order to obtain a DM estimate for each epoch while analyzing the GMRT data. Observation frequencies for the NANOGrav data can be seen in Figure 3 for comparison. GMRT data could provide low-frequency coverage in complement with the NANOGrav data and fill in the gaps where data below the $820 \mathrm{MHz}$ band do not exist in the 11 yr data set.

\section{DM Prediction and Comparison}

Observing at widely spaced frequencies can decrease DM estimation errors, thus increasing DM precision. This more precisely measured DM can then be used to calculate the corrected infinite-frequency TOA. Assuming there are no other chromatic timing perturbations, Cordes et al. (2016) show that if we observe at two widely separated frequencies $\nu_{1}$ and $\nu_{2}$ (where $\nu_{2}>\nu_{1}$ ) with corresponding average TOA uncertainties $\sigma_{1}$ and $\sigma_{2}$, the DM uncertainty can be expressed by

$$
\sigma_{\mathrm{DM}}=\frac{\sqrt{\sigma_{1}^{2}+\sigma_{2}^{2}}}{K\left(\nu_{1}^{-2}-\nu_{2}^{-2}\right)},
$$

which requires knowledge of the profile alignment across potentially disparate frequency bands. When evaluating the estimated DM uncertainty from a single observing band measurement centered at $\nu_{0}$, Equation (3) can be approximated by

$$
\sigma_{\mathrm{DM}} \approx \sqrt{2} \sigma_{\mathrm{TOA}} \frac{\nu_{0}^{3}}{K \Delta \nu},
$$

where $\Delta \nu$ is the observing bandwidth and $\sigma_{\mathrm{TOA}}$ is the average TOA uncertainty for the single band. The increase in DM precision with widely spaced frequencies (as opposed to using sub-bands across the bandwidth of one observing frequency) can be estimated for the inclusion of lower-frequency data via these two relations. When $\Delta \nu$ is considerably smaller than the difference between frequency bands, it can be seen that the quantity in Equation (3) is typically much smaller than that in Equation (4).

However, Cordes et al. (2016) also show that the DM itself is frequency-dependent (chromatic) even at a single epoch due to multipath scattering; calculating DM at different frequencies will result in different DM estimates because of the net difference in dispersive time delays. This in turn increases the uncertainties in DM estimation over wide bandwidths.

In order to determine the change in DM precision when using multifrequency observations in comparison with single-frequency observations, we need to examine how the TOA uncertainty changes between bands. The mean TOA uncertainty (i.e., averaged over all pulsars) at $1.4 \mathrm{GHz}$ for the NANOGrav $11 \mathrm{yr}$ data is $\sigma_{1.4 \mathrm{GHz}} \approx 600 \mathrm{~ns}$; following Equation (4) for an 

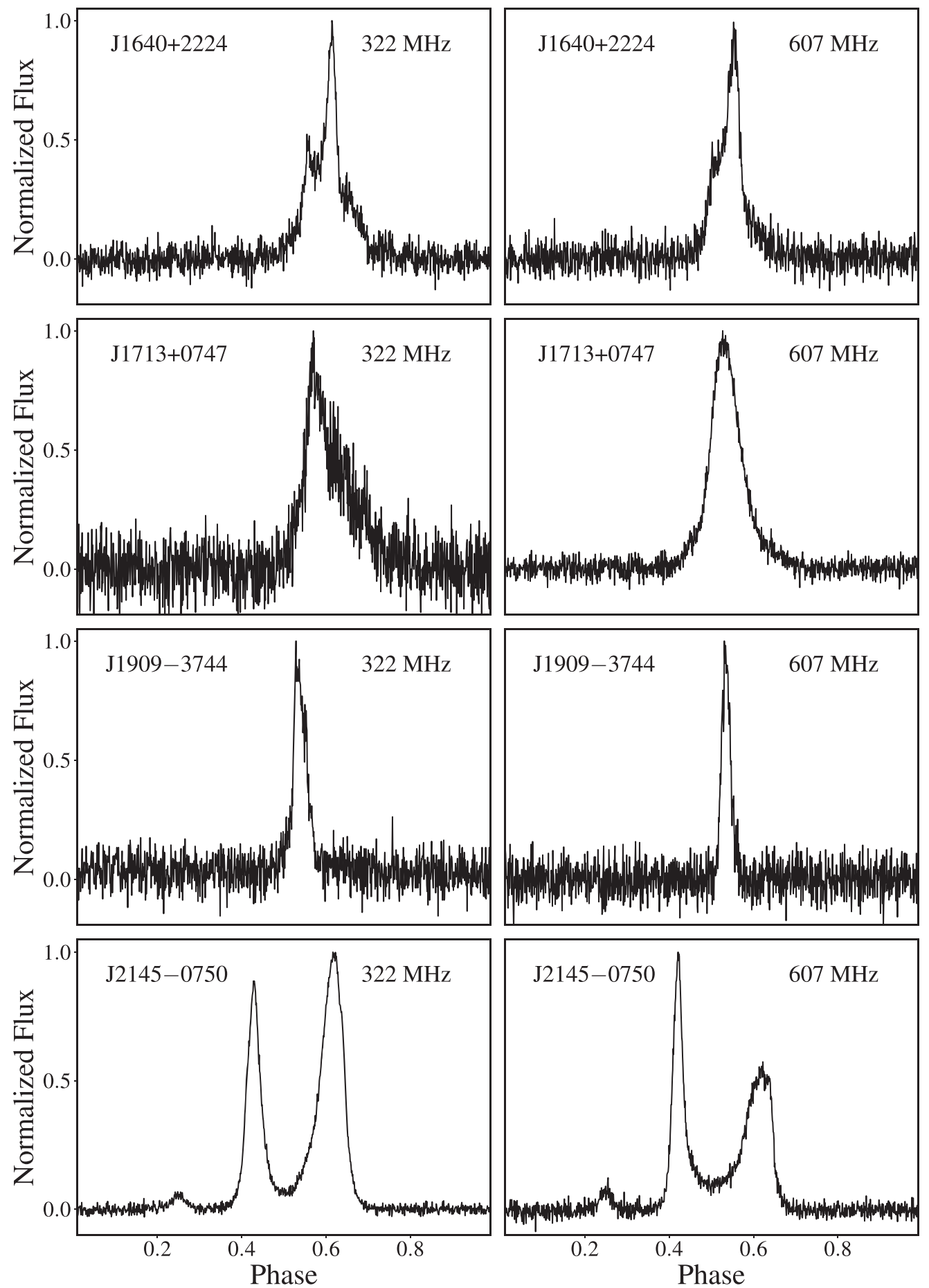

Figure 2. Sample pulse profiles for four MSPs from our GMRT observations. We show example single-epoch pulse profiles at $322 \mathrm{MHz}$ (left) and $607 \mathrm{MHz}$ (right) for PSRs J1640+2224, J1713+0747, J1909-3744, and J2145-0750 from top to bottom.

observation at $1.4 \mathrm{GHz}$, this yields a DM uncertainty of $\sigma_{\mathrm{DM}}=7 \times 10^{-4} \mathrm{pc} \mathrm{cm}^{-3}$ for a typical $800 \mathrm{MHz}$ bandwidth. To achieve the same DM uncertainty through our GMRT observations (where $\Delta \nu=32 \mathrm{MHz}$ ), we would require $\sigma_{322 \mathrm{MHz}}=2 \mu \mathrm{s}$ and $\sigma_{607 \mathrm{MHz}}=0.3 \mu \mathrm{s}$. These uncertainties are smaller than those we were generally able to achieve. However, when combining the two GMRT bands following Equation (3), the lower limit uncertainties jump to $\sigma_{322 \mathrm{MHz}} \approx 24 \mu \mathrm{s}$ and $\sigma_{607 \mathrm{MHz}} \approx 3.6 \mu \mathrm{s}$ assuming a similar scaling between the two bands as seen between the single TOA uncertainties (which may or may not be the case). Adding $1.4 \mathrm{GHz}$ data, the minimum TOA uncertainty requirements increase even higher to $\sigma_{322 \mathrm{MHz}}=30 \mu \mathrm{s}$ and $\sigma_{607 \mathrm{MHz}}=7.4 \mu \mathrm{s}$.

More generally, we can examine how TOA uncertainties change with frequency. We do this for the NANOGrav $11 \mathrm{yr}$ data set (Figure 3), highlighting the sources with GMRT observations. In general the lower-frequency bands have higher TOA uncertainties. Typical changes are a factor of $<2$ between 400 and $1400 \mathrm{MHz}$. This would imply that using Equation (3) will result in significantly smaller uncertainties in DM, and therefore more precise DM measurements, from combining the multiple frequencies than either frequency alone. 


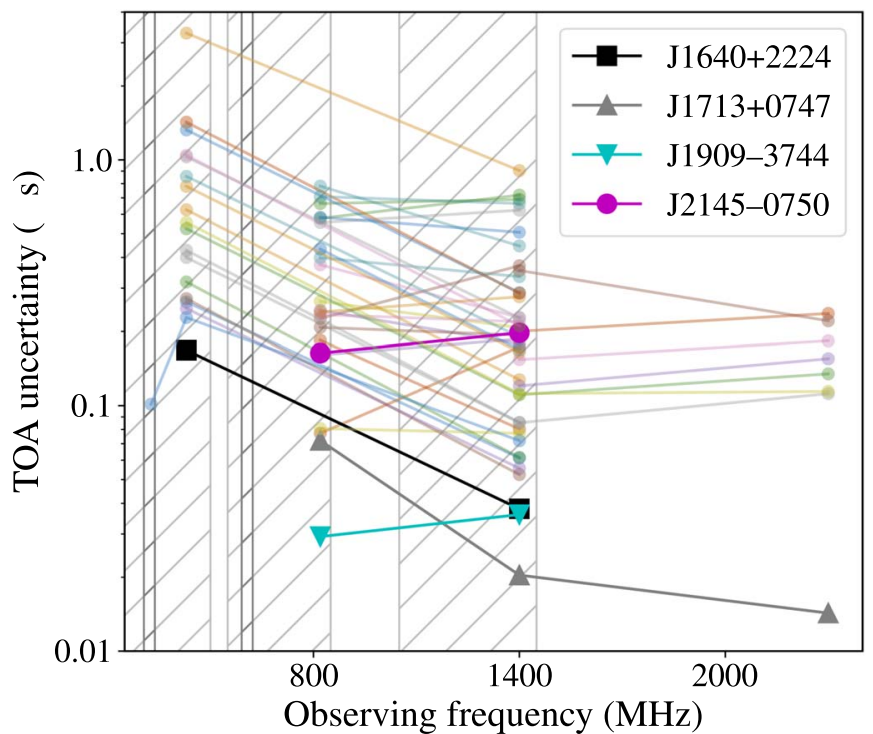

Figure 3. Average TOA uncertainties in the NANOGrav $11 \mathrm{yr}$ data set at various center frequencies (Arzoumanian et al. 2018). The MSPs with GMRT data are the labeled symbols. The lighter gray hatched regions show the potential GMRT observing frequencies, and the darker gray regions show the two frequencies used in this work. Note that the regions showing the potential observing ranges reflect the current bandwidth capabilities of the GMRT, which have since been upgraded from the $32 \mathrm{MHz}$ bandwidth available at the time of these observations (Gupta et al. 2017).

\subsection{Timing Results}

At each epoch, DMs were measured at $322 \mathrm{MHz}, 607 \mathrm{MHz}$, and then fit jointly using both frequencies and assuming no frequency evolution of the pulse profile. The DMs measured from these data are plotted alongside the NANOGrav DMs in Figure 4; overall, we see that the GMRT-measured DMs (both single frequency and combined) have significantly larger uncertainties than the NANOGrav-measured DMs. The singleepoch DMs measured here agree with the NANOGrav values for PSR J2145-0750 for all observing epochs and for most epochs for PSR J1909-3744. None of the DMs for J1640+2224 and $\mathrm{J} 1713+0747$ agree with the NANOGrav values; the DMs measured for $\mathrm{J} 1640+2224$ are consistently much smaller than the NANOGrav DMs by $\sim 0.03 \mathrm{pc} \mathrm{cm}^{-3}$, while DMs measured for PSR J1713+0747 are higher than the NANOGrav values by $\sim 0.07 \mathrm{pc} \mathrm{cm}^{-3}$. This is likely a result of unmodeled pulse profile evolution in the two GMRT frequency bands due to the resulting frequency-dependent biases (quantified by FD parameters in the timing model) being held fixed at the NANOGrav values; an independent fit for FD parameters could not be done here due to the small number of observing epochs. A more thorough description of NANOGrav FD parameters can be seen in Arzoumanian et al. (2015). The average DMs measured across all epochs can be seen in Table 1.

There are several reasons why DM measurements would differ between the two data sets in addition to pulse profile changes. Nonsimultaneous measurements between the different observatories could cause discrepancies. Some of the singleepoch DM measurements have nearly week-long differences between the NANOGrav and GMRT observing epochs. However, Jones et al. (2017) calculate the timescales it takes for the DM to vary beyond the measurement errors; the DM variation timescales for the pulsars timed here are all greater than 1 month, so this is unlikely to be the reason for the
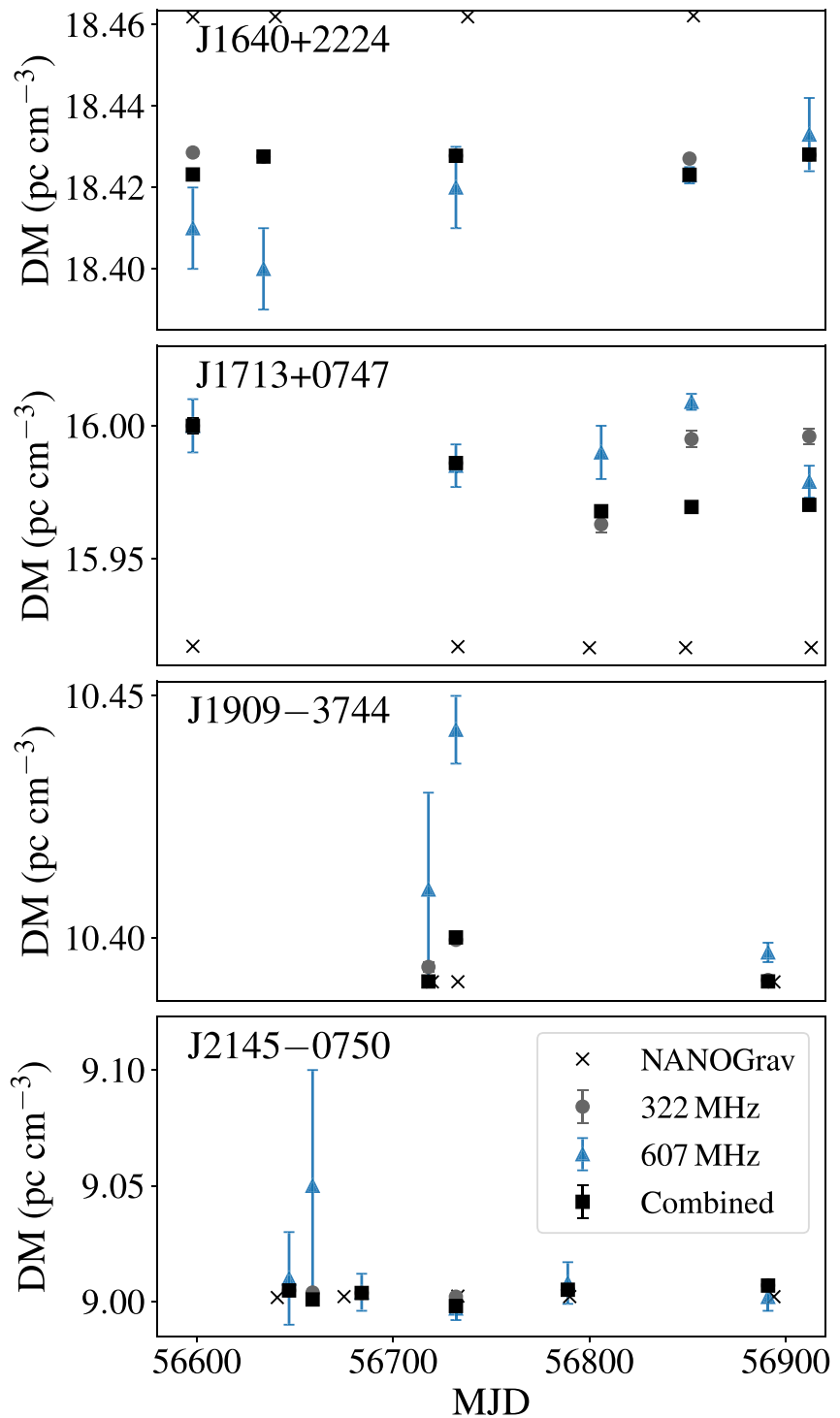

Figure 4. Comparison of single-epoch DMs for GMRT and NANOGrav $11 \mathrm{yr}$ measurements for the four MSPs considered here: PSRs J1640+2224, J1713 +0747 , J1909-3744, and J2145-0750 from top to bottom. GMRT timing was done using just the $322 \mathrm{MHz}$ data (circles), just the $607 \mathrm{MHz}$ data (triangles), and the two frequencies combined (squares).

Table 1

DM Estimates from Daily Timing

\begin{tabular}{lccccc}
\hline \hline PSR & $\begin{array}{c}\mathrm{DM}_{322} \\
\left(\mathrm{pc} \mathrm{cm}^{-3}\right)\end{array}$ & $\begin{array}{c}\sigma_{322} \\
(\mu \mathrm{s})\end{array}$ & $\begin{array}{c}\mathrm{DM}_{607} \\
\left(\mathrm{pc} \mathrm{cm}^{-3}\right)\end{array}$ & $\begin{array}{c}\sigma_{607} \\
(\mu \mathrm{s})\end{array}$ & $\begin{array}{c}\mathrm{DM}_{\text {total }} \\
\left(\mathrm{pc} \mathrm{cm}^{-3}\right)\end{array}$ \\
\hline $\mathrm{J} 1640+2224$ & $18.4281(4)$ & 1.7 & $18.426(2)$ & 2.9 & $18.42816(3)$ \\
$\mathrm{J} 1713+0747$ & $15.9888(9)$ & 8.9 & $16.002(3)$ & 3.0 & $15.98936(8)$ \\
$\mathrm{J} 1909-3744$ & $10.3945(3)$ & 2.8 & $10.407(2)$ & 3.6 & $10.459(2)$ \\
$\mathrm{J} 2145-0750$ & $9.0042(2)$ & 1.5 & $9.012(8)$ & 4.2 & $9.00453(3)$ \\
\hline
\end{tabular}

Note. Results from timing using the GMRT data. Columns list the average DM and average TOA error across all epochs for the 322 and $607 \mathrm{MHz}$ data, respectively, and the DM from timing using both frequencies bands. Quantities in parentheses are $1 \sigma$ uncertainties on the last digit.

discrepancies. Lam et al. (2016) modeled ionospheric DM variations and placed an upper limit to their DM contribution of $\sim 10^{-4} \mathrm{pc} \mathrm{cm}^{-3}$, two orders of magnitude smaller than all of 
Table 2

GMRT TOA Uncertainties and Predicted DM Precision

\begin{tabular}{|c|c|c|c|c|c|c|}
\hline PSR & $\begin{array}{l}\sigma_{322} \\
(\mu \mathrm{s})\end{array}$ & $\begin{array}{l}\sigma_{607} \\
(\mu \mathrm{s})\end{array}$ & $\begin{array}{l}\sigma_{\mathrm{DM} ; \mathrm{GMRT}} \\
\left(\mathrm{pc} \mathrm{cm}^{-3}\right)\end{array}$ & $\begin{array}{c}\sigma_{\mathrm{DM} ; \mathrm{NG}} \\
\left(\mathrm{pc} \mathrm{cm}^{-3}\right)\end{array}$ & $\begin{array}{c}\sigma_{322 ; \text { target }} \\
(\mu \mathrm{s})\end{array}$ & $\begin{array}{c}\sigma_{607 ; \text { target }} \\
(\mu \mathrm{s})\end{array}$ \\
\hline $\mathrm{J} 1640+2224$ & $0.59-4.3$ & $0.75-5.7$ & $>1.5 \times 10^{-5}$ & $9 \times 10^{-6}$ & $<0.4$ & $<0.07$ \\
\hline $\mathrm{J} 1713+0747$ & $3.1-12.7$ & $1.1-5.4$ & $>8 \times 10^{-5}$ & $9 \times 10^{-6}$ & $<0.4$ & $<0.1$ \\
\hline J1909-3744 & $0.8-4.5$ & $0.7-12$ & $>2 \times 10^{-5}$ & $1 \times 10^{-5}$ & $<1.2$ & $<0.4$ \\
\hline $\mathrm{J} 2145-0750$ & $0.71-2.4$ & $1.2-12$ & $>2 \times 10^{-5}$ & $5 \times 10^{-5}$ & $<2.0$ & $<0.4$ \\
\hline
\end{tabular}

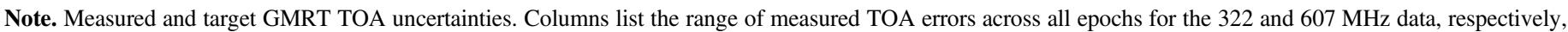

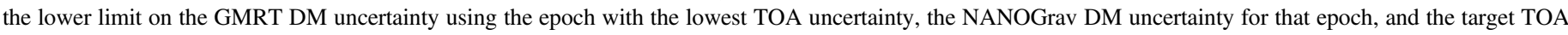
uncertainties needed to reach the NANOGrav DM precision.

the DM differences seen in Figure 4. Following Equation (12) in Cordes et al. (2016), which assumes scattering is due to a thin screen, a fiducial pulsar observed at 322 and $607 \mathrm{MHz}$ would result in an rms DM offset due to chromatic DMs of $\sim 10^{-4} \mathrm{pc} \mathrm{cm}^{-3}$. Correcting only for DM without correcting for scattering will cause discrepancies as the DM fit will absorb some scattering effects; however, all four MSPs have DMs below $20 \mathrm{pc} \mathrm{cm}^{-3}$, so they likely do not show sufficient amounts of scattering to be absorbed in the DM modeling. Hence, none of these mechanisms are sufficient to explain the scale of the DM offsets we measure.

Since these observations were taken, the GMRT has undergone system improvements to create the UGMRT, including wider observing bandwidth capabilities and more sensitive receiver systems (Gupta et al. 2017). The uGMRT has a maximum instantaneous bandwidth of $400 \mathrm{MHz}$; usable bandwidths at the observing frequencies used here are predicted to be 120-200 MHz. The comparable uGMRT observing bands to those used here are centered at 400 and $650 \mathrm{MHz}$.

Table 2 shows the lower limits on the DM precision for each MSP based on the measured TOA uncertainties, as well as the predicted upper limit on TOA uncertainties for future observations required to match the precision of NANOGravmeasured DMs. PSR J2145-0750 is the only source timed here for which adding in the GMRT $322 \mathrm{MHz}$ data in their current state improve the uncertainty on DM. The necessary precision for this MSP could be achieved at $650 \mathrm{MHz}$ with the uGMRT with a slightly longer ( $\sim 40$ minutes) observation at each epoch using half the array. For $\mathrm{J} 1640+2224$ and J1909-3744, the necessary precision could be reached at $400 \mathrm{MHz}$ using a similar observing strategy with the uGMRT and, in the case of J1909-3744, at $650 \mathrm{MHz}$ as well; J1640 +2224 would require a more robust observing strategy $(\sim 2 \mathrm{hr}$ per epoch with the full array) at $650 \mathrm{MHz}$. Observations of $\mathrm{J} 1713+0747$ could achieve the minimum precision at $400 \mathrm{MHz}$ by observing for $2 \mathrm{hr}$ per epoch with half of the array, and at $650 \mathrm{MHz}$ observing for $\sim 2.5 \mathrm{hr}$ per epoch using the full array.

\section{Baseline Ripple}

Separate from the ISM effects discussed above, additional telescope-specific effects can reduce TOA precision. In particular, low temporal-frequency (i.e., red) noise in pulsar profiles can systematically pull a TOA to an earlier or later time, and appears as a stochastic contribution to the TOA error budget. We call this baseline ripple, and it could be due to radiofrequency interference, typically due to a nearby power line or other likely epoch-dependent effects. The phase of the ripple is random relative to the pulse, and therefore is more noticeable for canonical pulsars than MSPs due to the smaller number of times the data are folded over the pulse period (which will be different than the ripple period). An example of baseline ripple seen in the GMRT data for our test pulsar B1929+10 can be seen in Figure 5. While it may not be noticeable by eye for MSPs, it is important that we estimate the effect of baseline ripple on precision MSP timing.

We define a data profile $I(t)$ that is composed of the pulse template $T(t)$ with pulse amplitude $A$ added to a sinusoidal baseline ripple with amplitude $r$, phase $\phi$, and frequency $f_{r}=\omega_{r} / 2 \pi=1 / P_{r}$,

$$
I(t)=A \times T\left(t-t_{0}\right)+r \cos \left[\omega_{r}\left(t-t_{0}\right)+\phi\right],
$$

where $t_{0}$ is an arbitrary reference time. For a Gaussian pulse with FWHM $W$, the template pulse template can be modeled as

$$
T(t)=e^{-4 \ln 2(t / W)^{2}},
$$

where the approximate error on the TOA becomes

$$
\frac{\sigma_{\mathrm{TOA}}}{W}=\left(\frac{\pi}{4 \ln 2}\right)\left(\frac{r}{A}\right)\left(\frac{W}{P_{r}}\right) e^{-4 \pi \ln 2\left(W / P_{r}\right)^{2}} .
$$

This derivation is discussed in Appendix A.

Because the ripple and pulse periods are likely nonharmonic, we use a Lomb-Scargle periodogram to search for any latent periodicity. Applying a Lomb-Scargle periodogram to the pulse profile for our test pulsar PSR B1929+10, we detect a ripple frequency of $f_{r}=52 \pm 5 \mathrm{~Hz}$, seen in Figure 6. This matches with the AC power-line frequency in India $(50 \mathrm{~Hz})$. Epochs where the detected ripple was below a $3 \sigma$ significance threshold were not included in this estimate; only one epoch did not show a detectable ripple $(<1 \sigma$ peak). The induced timing errors due to ripple for the four MSPs can be estimated via Equation (7) using our observations of PSR B1929+10 to measure the amplitude $r$ of the baseline ripple. Scaling the detected ripple amplitude to the MSP flux densities relative to B1929+10 at each epoch, we estimate $r / A \approx 0.03$ in our MSP observations. With the respective pulse widths and periods, this signal can cause timing uncertainties up to $\sim 150 \mathrm{~ns}$ for J1640 +2224 and $\mathrm{J} 1909-3744$, up to $~ 340 \mathrm{~ns}$ for $\mathrm{J} 1713+0747$, and up to a microsecond for J2145-0750 (due to its longer period and larger pulse width). Given that the achievable GMRT timing precisions in this work are a few microseconds or greater, baseline ripple does not appear to be a concern for these data (but a similar signal could be important for NANOGrav data taken with the GBT). This effect will need 


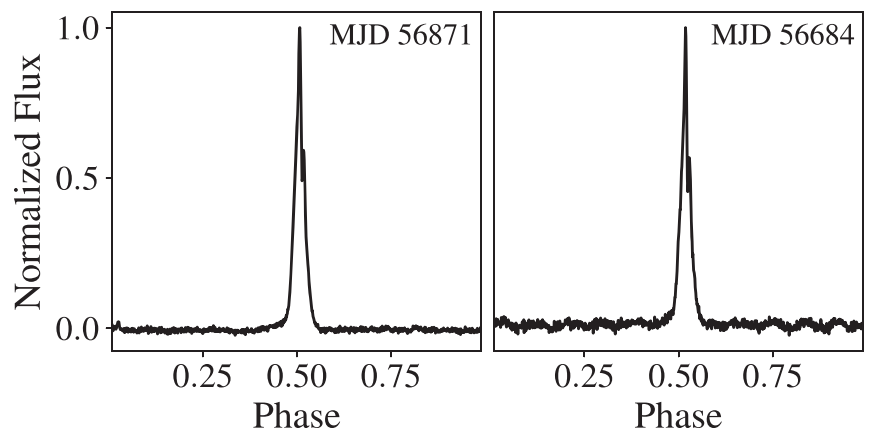

Figure 5. Pulse profile for PSR B1929+10 observed with the GMRT at $607 \mathrm{MHz}$ on two different epochs separated by about 2 weeks. The left profile does not show a visible level of baseline ripple, whereas the right profile shows clearly evident ripple in the baseline.
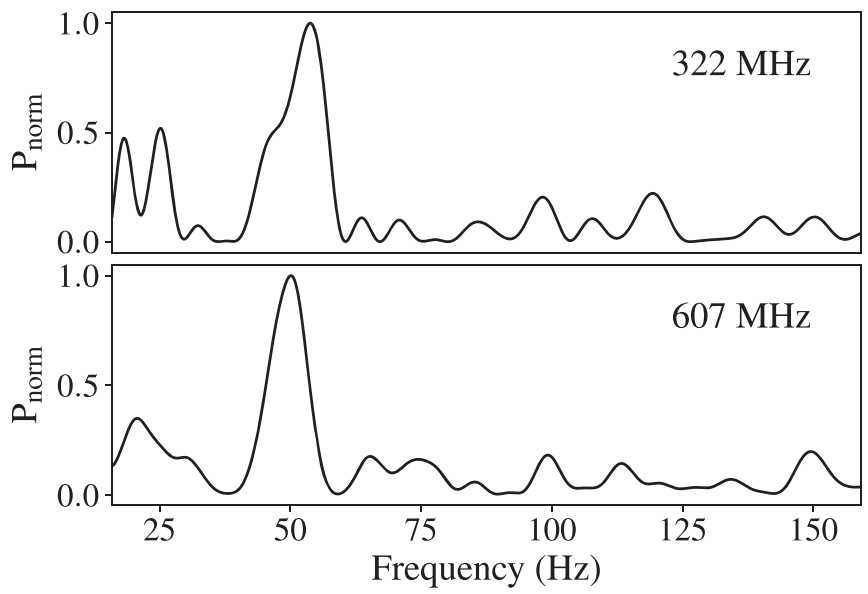

Figure 6. Lomb-Scargle periodograms for the baseline ripple apparent in in PSR B1929+10; the profile can be seen in Figure 5. The power is in arbitrary units. The periodogram shows a significant peak $(\sim 3 \sigma-6 \sigma$ depending on the epoch) at approximately $52 \mathrm{~Hz}$. Epochs that showed peaks below $3 \sigma$ significance were not included in this analysis.

to be considered for high-precision pulsar timing observations with the uGMRT.

\section{Interstellar Scintillation}

In addition to timing analyses, measurements of the modulated radio pulsar emission due to scintillation in the ISM can directly probe the properties of the intervening material. Scintillation bandwidths can be used to estimate delays from pulse broadening on the TOAs and can therefore help to track scattering delays and discriminate between those and DM delays. Scintillation observations do not require the timing accuracy or precision of pulsar timing experiments. Measurements of scintles, intensity maxima in a dynamic spectrum $I(t, \nu)$, require high $\mathrm{S} / \mathrm{N}$ pulse profiles observed over sufficiently large bandwidths to capture the maxima, but also high frequency and time resolution to discriminate individual scintles. The characteristic bandwidth and timescale of scintles vary as different functions of frequency, with both becoming smaller at lower radio frequencies. GMRT observations at low frequencies can therefore extract useful information about ISM, in particular for the lowest DM pulsars with the largest scintle sizes.

The standard procedure for measuring the scintillation parameters, the characteristic bandwidth $\Delta \nu_{\mathrm{d}}$ and timescale

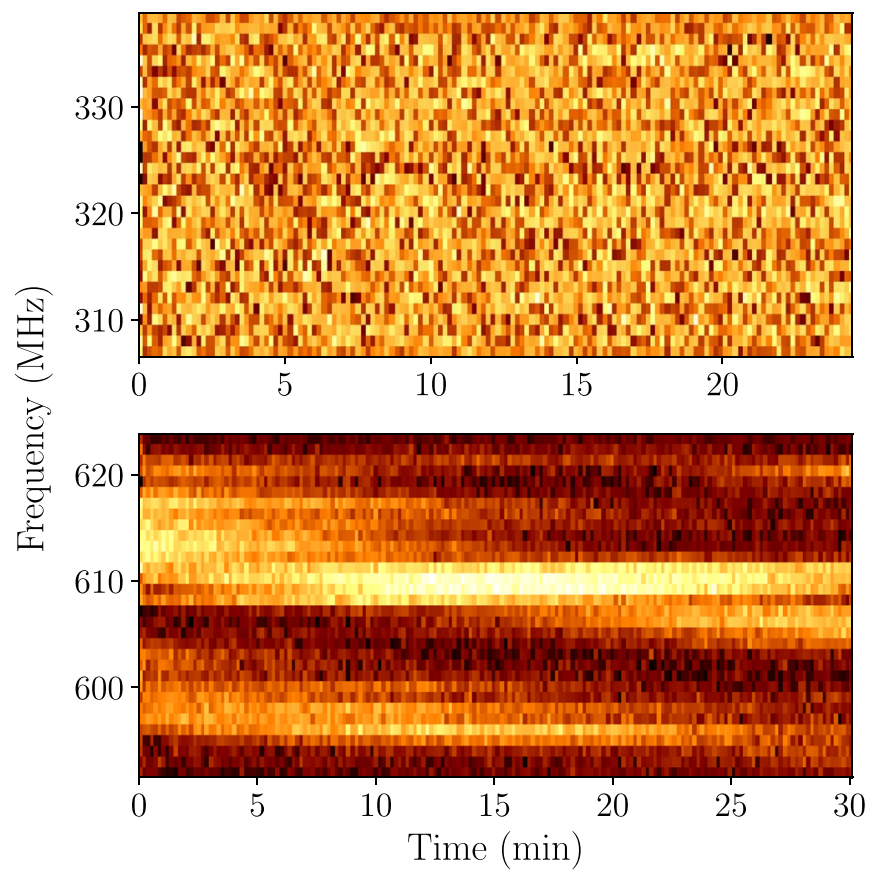

Figure 7. Dynamic spectra for PSR J1713+0747 on MJD 56852. The scintles can be clearly seen in the $600 \mathrm{MHz}$ band. Note that the total time spanned by the observation may vary by several minutes between the two bands.

$\Delta t_{\mathrm{d}}$, from the dynamic spectrum is via an autocorrelation function $(\mathrm{ACF})$. For a dynamic spectrum $I(t, \nu)$, we define the 2D ACF as

$$
R_{I}(\delta t, \delta \nu)=\langle I(t, \nu) I(t+\delta t, \nu+\delta \nu)\rangle .
$$

In the case when the $\mathrm{S} / \mathrm{N}$ values of ACFs determined for each epoch are low, we can take the ACFs and add them together to increase the total $\mathrm{S} / \mathrm{N}$ with which to measure scintillation parameters:

$$
R_{I}(\delta t, \delta \nu)=\sum_{n} R_{I, n}(\delta t, \delta \nu)
$$

where $n$ is the index over the epoch. This procedure assumes that variations in the scintillation parameters are small relative to the uncertainties. While single-epoch ACFs typically had low $\mathrm{S} / \mathrm{N}$, we were able to measure average scintillation parameters across all data sets with an increased $\mathrm{S} / \mathrm{N}$. Using PYPULSE $^{14}$ (Lam 2017), we generated ACFs for epochs when distinct scintles were seen. ACFs were added together based on the appearance of scintles in the band, which enabled more robust measurements of the scintillation bandwidths. Dynamic spectra produced for PSRs J1713+0747 and J2145-0750 can be seen in Figures 7 and 8.

For PSR J1713+0747, we co-added ACFs from five epochs of the $607 \mathrm{MHz}$ data $(56806,56852,56912,56598$, and 56955), again when distinct scintles were seen. For PSR J2145-0750, we co-added ACFs from two epochs of $322 \mathrm{MHz}$ data (56732 and 56891). We fit a 1D Gaussian to the slice of the 2D ACF at zero time lag, i.e., $R_{I}(0, \delta \nu)$, and calculated the half-width at half maximum as is standard (e.g., Cordes 2002). For PSR J1713 +0747 , we measured $\Delta \nu_{\mathrm{d}, 607}=3.1 \mathrm{MHz}$, and for PSR J2145$0750 \Delta \nu_{\mathrm{d}, 322}=3.6 \mathrm{MHz}$. Scintillation bandwidth scales with

\footnotetext{
${ }^{14}$ https://github.com/mtlam/PyPulse
} 


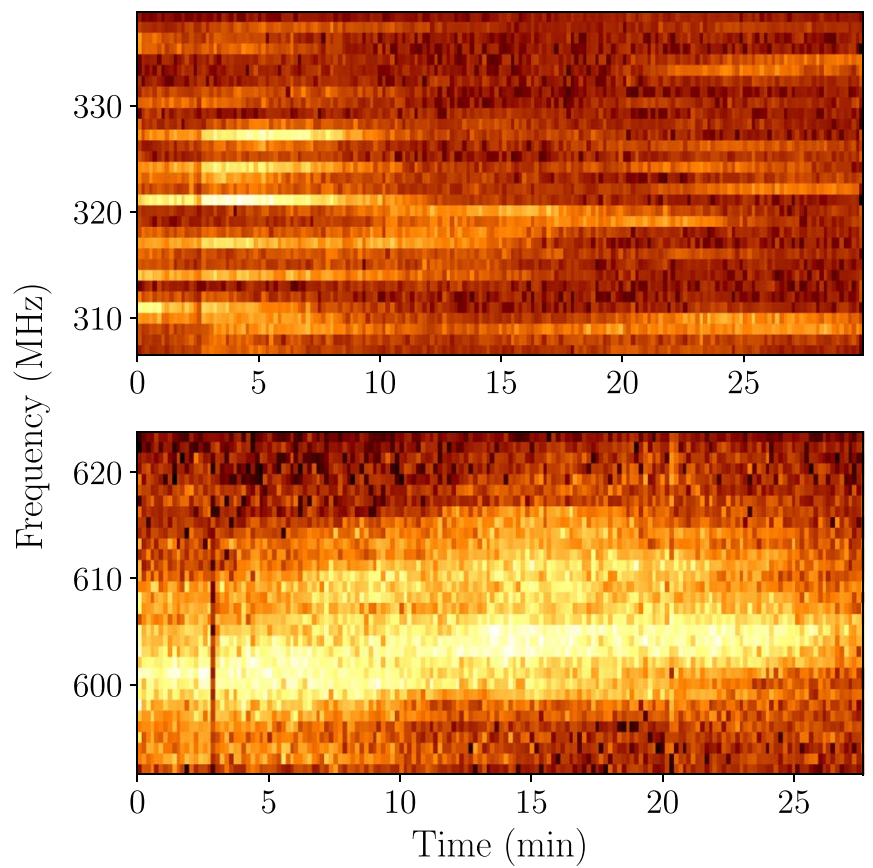

Figure 8. Dynamic spectra for PSR J2145-0750 on MJD 56732. The scintles can be clearly seen in both the 300 and $600 \mathrm{MHz}$ bands. Note that the total time spanned by the observation may vary by several minutes between the two bands.

observing frequency as

$$
\Delta \nu_{\mathrm{d}}=\Delta \nu_{0}\left(\frac{\nu}{\nu_{0}}\right)^{\xi},
$$

where $\nu$ and $\nu_{0}$ are the higher and lower observing frequencies, $\Delta \nu_{\mathrm{d}}$ and $\Delta \nu_{0}$ are the higher and lower scintillation bandwidths, and $\xi$ is the scaling factor. Analysis of the NANOGrav $11 \mathrm{yr}$ data set found $\Delta \nu_{\mathrm{d}, 1400}=21.1 \pm 8.6 \mathrm{MHz}$ and $47.8 \pm 13.3 \mathrm{MHz}$ at $\nu=1400 \mathrm{MHz}$ for $\mathrm{J} 1713+0747$ and $\mathrm{J} 2145-0750$, respectively (Levin et al. 2016). Comparing these two measurements corresponds to a scaling factor of $\xi=2.3 \pm 0.5$ for $\mathrm{J} 1713$ +0747 and $\xi=1.8 \pm 0.2$ for $\mathrm{J} 2145-0750$. The scintillation bandwidth is expected to scale in frequency as $\nu^{22 / 5}$ for a Kolmogorov medium with a single thin scattering screen (Cordes \& Lazio 2002). Frequency-dependent scintillation bandwidth scaling shallower than $22 / 5$ is not unexpected and has been seen in other analyses (e.g., Bhat et al. 2004; Levin et al. 2016; Turner et al. 2020). A possible explanation for the discrepancy between the measurements is that there are two scintillation scales. Finding multiple scales (within an order of magnitude) of scintillation is a known effect for nearby pulsars (Gwinn et al. 2006). Longer-term monitoring of the scintillation parameters at lower frequencies will be required to make more definitive claims regarding these discrepancies. Note that we should treat these single-epoch scintillation measurements with caution, as it is well known that the scintillation bandwidth can vary dramatically from epoch to epoch (Coles et al. 2015).

The scintillation bandwidth can be used to calculated the scattering delay $\tau_{\mathrm{d}}=C_{1} / 2 \pi \Delta \nu_{\mathrm{d}}$, where $C_{1}$ is a constant that varies with the geometry and spectral model of the ISM; we adopt $C_{1}=0.654$, which corresponds to a Kolmogorov medium with a thin scattering screen (Lambert \& Rickett 1999).
This yields a scattering delay of $\tau_{\mathrm{d}} \approx 30 \mathrm{~ns}$ for both pulsars. These delays are approximately two orders of magnitude smaller than the TOA errors we measure here and therefore are not an issue in these data. However scattering correction may become important for low-frequency observations used for GW detection where the goal is $\lesssim 100 \mathrm{~ns}$ timing precision.

The transverse velocity of the pulsar can be estimated using the scintillation timescale

$$
V_{\mathrm{ISS}}=A_{\mathrm{ISS}} \frac{\sqrt{\Delta \nu_{\mathrm{d}} D_{\mathrm{kpc}} x}}{\nu_{\mathrm{GHz}} \Delta \tau_{\mathrm{d}}},
$$

where $A_{\text {ISS }}=2.53 \times 10^{4} \mathrm{~km} \mathrm{~s}^{-1}$ for a Kolmogorov medium and $x=D_{o} / D_{p}$, where $D_{o}$ is the distance between the screen and the observer and $D_{p}$ is the distance between the screen and the pulsar (Gupta et al. 1994; Cordes \& Rickett 1998; Turner et al. 2020); we assume $x=1$ for a screen halfway along the LOS. Here, we use a lower limit equal to the length of our observations $\Delta \tau_{\mathrm{d}} \geqslant 30$ minutes, which was not long enough to characterize the scintillation timescale. We can therefore calculate upper limits of the transverse velocity of $V_{\text {ISS }}<43 \mathrm{~km} \mathrm{~s}^{-1}$ and $V_{\text {ISS }}<74 \mathrm{kms}^{-1}$ for for $\mathrm{J} 1713+0747$ and $\mathrm{J} 2145-0750$, respectively; these agree with published velocities derived through proper motion measurements (Turner et al. 2020).

\section{Conclusions}

This work illustrates the one of the first attempts to utilize the GMRT for IPTA work. We have demonstrated that the GMRT can be successfully used to time MSPs and measure DM, in some cases with comparable sensitivity to current PTA data. We observed with only a subset of the array for the data presented here; one factor that would go a long way in improving data precision is using the complete array for timing observations. With the availability of larger instantaneous bandwidth (e.g., 300-500 MHz) for the uGMRT, the similar observations for monitoring the ISM effects can be carried out with the complete array. The GMRT observer's manual ${ }^{15}$ predicts the array gain as $\sim 0.33 \mathrm{~K} \mathrm{Jy}^{-1}$ antenna ${ }^{-1}$; if the entire array were used (double the maximum number of antennas used here), the predicted gain increases to $\sim 10 \mathrm{~K} \mathrm{Jy}^{-1}$, assuming no losses due to beam forming. For comparison, the Arecibo Observatory lists a gain of $11 \mathrm{~K} \mathrm{Jy}^{-1}$ at similar frequencies.

For all of the MSPs discussed above, known DM effects could not account for the offset seen between single-epoch DM measurements, which suggests that the sources of these variations are not due to the ISM. Due to chromatic DMs, we would not expect agreement between DMs measured at different frequencies, but as discussed above the measured differences are larger than can be explained by chromatic DMs. DMs may show variability due to a combination of scintillation and pulse profile evolution, as we essentially see a different part of the pulse at each epoch. The NANOGrav data set includes FD parameters in the timing model, which account for pulse profile evolution, while this analysis did not due to the limited number of observing epochs. This combination is the likely source of a DM offset between frequencies. ${ }^{16}$

\footnotetext{
15 http://www.gmrt.ncra.tifr.res.in/

16 After the submission of this work, the uGMRT has seen similar DM offsets to NANOGrav DMs; these offsets are also attributed to variations in pulse profile templates (Krishnakumar et al. 2021).
} 
As mentioned in Section 2, polarization calibration was not done when the GMRT data used here were obtained. Not accounting for polarization causes TOA uncertainties due to deviations from the pulse profile template. Using fiducial values for NANOGrav data, the anticipated TOA uncertainty induced by errors in polarization calibration to be $\sim 100 \mathrm{~ns}-$ $1 \mu$ s (van Straten 2006; Lam et al. 2018) for narrow frequency channels (this value averages down when integrating in time, but may change systematically between epochs); as no polarization calibration was performed, the errors for the GMRT data are likely larger. Without significant standardizations in high-fidelity polarization calibration, TOAs obtained by the uGMRT system will be difficult to integrate into global PTA efforts.

While the data presented here overall do not meet the required TOA uncertainties for an improved DM measurement, high-precision timing required by PTAs appears feasible with the upgraded capabilities of the uGMRT. This sensitivity should be achievable with a similar observing strategy used here, and in some cases with longer observation times and larger subsets of the array. Even without PTA-level timing precision, the lower-frequency timing data of the GMRT will still provide valuable science related to the ISM and propagation effects. Lower-frequency data show smaller scintles than at higher frequencies; the increased bandwidth of the uGMRT systems will effectively capture more scintles and greatly boost the $\mathrm{S} / \mathrm{N}$ of ACF measurements. Given the smaller scintle sizes, the uGMRT should prioritize short subintegrations and small frequency-channel resolutions in their observations. While costly in terms of data volume, it is possible to develop real-time pipelines to save these data products. Measurements of dynamic spectra over long time spans are critical in helping to constrain the properties of the turbulent ionized ISM. In turn, even without high-precision TOAs, these constraints can feed into PTA analyses, allowing for improved mitigation of scattering effects among all pulsars in the array. The high-precision DMs provided by the uGMRT could play a very important role in IPTA data sets going forward, especially giving the recent loss of the Arecibo telescope.

The NANOGrav Collaboration is supported by NSF Physics Frontier Center award \#1430284. M.T.L. also acknowledges support from NSF AAG award number 2009468. We acknowledge support of the Department of Atomic Energy, Government of India, under project number 12-R\&D-TFR-5.02-0700. The GMRT is run by the National Centre for Radio Astrophysics of the Tata Institute of Fundamental Research, India. We acknowledge support of GMRT telescope operators for observations.

Facility: GMRT.

Software: DSPSR (van Straten \& Bailes 2010), PSRCHIVE (van Straten et al. 2011), PyPulse (Lam 2017), TEMPO (Nice et al. 2015).

\section{Appendix A TOA Offsets Due to Baseline Ripple}

Here, we derive the perturbations to pulse arrival times due to an unmodeled sinusoidal ripple. We define a data profile $I(t)$ that is composed of the pulse template $T(t)$ with amplitude $A$ and arrival time $t_{0}$, and some sinusoidal baseline ripple with amplitude $r$, phase $\phi$, and temporal frequency $f=\omega / 2 \pi=1 / P_{r}$,

$$
I(t)=A \times T\left(t-t_{0}\right)+r \cos \left[\omega\left(t-t_{0}\right)+\phi\right] .
$$

The template fitting procedure used to calculate the TOA can be represented in terms of finding the maximum crosscorrelation of the data $I$ and template $T, C_{I T}(\tau)$, the solution of which gives the TOA $\hat{\tau}$ :

$$
\begin{gathered}
C_{I T}(\tau)=\int d t I(t) T(t-\tau) \\
\Longrightarrow \frac{d C_{I T}}{d \tau}(\hat{\tau})=0 .
\end{gathered}
$$

We can expand around $T^{\prime}(t-\tau)$ about $T^{\prime}\left(t-t_{0}\right)$ to first order by assuming that the TOA error $\delta \tau=\hat{\tau}-t_{0}$ is much smaller that the template pulse width $W$. Doing this expansion and solving for the TOA error in Equation (A3) gives

$$
\delta \tau=\hat{\tau}-t_{0} \approx \frac{\int d t I(t) T^{\prime}(t)}{\int d t I(t) T^{\prime \prime}(t)}
$$

Plugging in Equation (A1) yields

$$
\delta \tau=-\frac{(r f / 2 \pi) \mathfrak{I}\left\{e^{-i \phi} \tilde{T}(f)\right\}}{A \int d f^{\prime} f^{\prime 2}\left|\tilde{T}\left(f^{\prime}\right)\right|^{2}+f^{2} r \Re\left\{e^{-i \phi} \tilde{T}(f)\right\}},
$$

where $\tilde{T}(f)$ if the Fourier transform of the template using $e^{-2 \pi i f t}$ and $\mathfrak{R}$ and $\mathfrak{I}$ are the real and imaginary parts, respectively.

For $r / A \ll 1$,

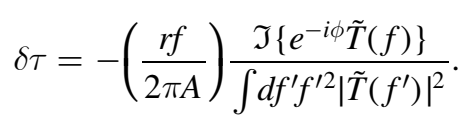

Multiple ripple terms then add linearly to the net TOA error; multiple sinusoids can be considered as a Fourier sum.

For a Gaussian pulse with FWHM $W$,

$$
T(t)=e^{-4 \ln 2(t / W)^{2}},
$$

the approximate error given by Equation (A6) becomes

$$
\frac{\delta \tau}{W}=\left(\frac{\pi}{4 \ln 2}\right)\left(\frac{r}{A}\right)\left(\frac{W}{P_{r}}\right) e^{-\pi^{2}\left(W / P_{r}\right)^{2} / 4 \ln 2} \sin \phi,
$$

which is used to estimate the ripple-induced TOA error in Section 4 (Equation (7)) The maximum of $\left(W / P_{r}\right) e^{-\pi\left(W / P_{r}\right)^{2} / 4 \ln 2}$ occurs at $W / P_{r}=\sqrt{2 \ln 2} / \pi=0.375$, corresponding to a maximum error

$$
\left(\frac{\delta \tau / W}{r(\sin \phi) / A}\right)=(8 e \ln 2)^{-1 / 2}=0.258 .
$$

For a multi-epoch data set, we expect $\phi$ to be uniformly distributed in $[0,2 \pi]$ yielding $\sigma_{\sin \phi}=1 / \sqrt{2}$. Then for $r=$ constant at all epochs, the maximum rms error is

$$
\begin{aligned}
\left(\frac{\sigma_{\delta \tau} / W}{r / A}\right)_{\max } & =\sigma_{\sin \phi}(8 e \ln 2)^{-1 / 2} \\
& =(4 \sqrt{e \ln 2})^{-1}=0.182 .
\end{aligned}
$$




\section{Appendix B Mean Amplitude of Folded Ripple Sinusoid}

Here we derive how the ripple amplitude changes, and therefore induced TOA offsets, when folding the ripple sinusoid over different pulse periods. We define a sinusoidal baseline ripple $x(t)=r \sin (\omega t)$. The time $t$ is related to the pulsar phase as $t=(m+\delta \theta) P$, where $m$ is an integer and the fractional phase $\delta \theta$ is between 0 and 1 . The ripple evaluated at the pulse phase is

$$
x(\delta \theta)_{m}=r \sin [\omega(m P+\delta \theta P)] .
$$

The profile of the baseline ripple averaged over a number of $M$ folded pulses is given by

$$
\begin{aligned}
S(\delta \theta) & =\frac{1}{M} \sum_{m=0}^{M-1} x(\delta \theta)_{m} \\
& =\frac{1}{M} \sum_{m=0}^{M-1} r e^{i(\omega m P+\omega \delta \theta P) .}
\end{aligned}
$$

Evaluating the sum shows how the ripple profile varies sinusoidally in the pulse phase

$$
S(\delta \theta)=e^{i \delta \theta P}\left(\frac{r \sin (\omega M P / 2)}{M \sin (\omega P / 2)}\right) .
$$

The term in parentheses is the amplitude of the ripple, so we can write

$$
|S|=\left|\frac{r \sin (\omega M P / 2)}{M \sin (\omega P / 2)}\right| .
$$

As $\omega=2 \pi / P_{r}$, we let the ratio of the spin period and ripple period $P / P_{r}=k+\delta \psi$, where $k$ is an integer (this assumes $P>P_{r}$, but this can easily be adapted to $P<P_{r}$ ). The ripple amplitude then becomes

$$
|S|=\left|\frac{r \sin (\pi M \delta \psi)}{M \sin (\pi \delta \psi)}\right| .
$$

When the two periods are harmonically related $(\delta \psi=0)$, the ripple amplitude does not decrease with folding. Otherwise the exact ripple amplitude depends on $\delta \psi$ (and is highest for $\delta \psi<1 / M$ ) but the expected value decreases as $1 / M$.

\section{ORCID iDs}

M. L. Jones (10 https://orcid.org/0000-0001-6607-3710

M. A. McLaughlin (1) https://orcid.org/0000-0001-7697-7422

J. Roy (i) https://orcid.org/0000-0002-2892-8025

M. T. Lam (1) https://orcid.org/0000-0003-0721-651X

J. M. Cordes (ㄷ) https://orcid.org/0000-0002-4049-1882

D. L. Kaplan (1) https://orcid.org/0000-0001-6295-2881

B. Bhattacharyya (1) https://orcid.org/0000-0002-6287-6900

L. Levin (1) https://orcid.org/0000-0002-2034-2986

\section{References}

Abbott, B. P., Abbott, R., Abbott, T. D., et al. 2016, PhRvL, 116, 061102 Arzoumanian, Z., Baker, P. T., Blumer, H., et al. 2020, ApJL, 905, L34 Arzoumanian, Z., Baker, P. T., Brazier, A., et al. 2018, ApJ, 859, 47 Arzoumanian, Z., Brazier, A., Burke-Spolaor, S., et al. 2015, ApJ, 813, 65 Bailes, M., Jameson, A., Abbate, F., et al. 2020, PASP, 37, e028 Bansal, K., Taylor, G. B., Stovall, K., \& Dowell, J. 2019, ApJ, 875, 146 Bhat, N. D. R., Cordes, J. M., Camilo, F., Nice, D. J., \& Lorimer, D. R. 2004, ApJ, 605, 759

Bhat, N. D. R., Tremblay, S. E., Kirsten, F., et al. 2018, ApJS, 238, 1

Bilous, A. V., Bondonneau, L., Kondratiev, V. I., et al. 2020, A\&A, 635, A75 Blasi, S., Brdar, V., \& Schmitz, K. 2020, PhRvR, 2, 043321

Buchmuller, W., Domcke, V., \& Schmitz, K. 2020, PhLB, 811, 135914 Coles, W. A., Kerr, M., Shannon, R. M., et al. 2015, ApJ, 808, 113

Cordes, J. M. 2002, in ASP Conf. Ser., 278, Single-Dish Radio Astronomy: Techniques and Applications, ed. S. Stanimirovic et al. (San Francisco, CA: ASP), 227

Cordes, J. M., \& Lazio, T. J. W. 2002, arXiv:astro-ph/0207156

Cordes, J. M., \& Rickett, B. J. 1998, ApJ, 507, 846

Cordes, J. M., Shannon, R. M., \& Stinebring, D. R. 2016, ApJ, 817, 16

Demorest, P. B., Ferdman, R. D., Gonzalez, M. E., et al. 2013, ApJ, 762, 94

Desvignes, G., Caballero, R. N., Lentati, L., et al. 2016, MNRAS, 458, 3341 Gupta, Y., Ajithkumar, B., Kale, H., et al. 2017, CSci, 113, 707

Gupta, Y., Rickett, B. J., \& Lyne, A. G. 1994, MNRAS, 269, 1035

Gwinn, C. R., Hirano, C., \& Boldyrev, S. 2006, A\&A, 453, 595

Hobbs, G., Dai, S., Manchester, R. N., et al. 2019, RAA, 19, 020

Isaacman, R., \& Rankin, J. M. 1977, ApJ, 214, 214

Jones, M. L., McLaughlin, M. A., Lam, M. T., et al. 2017, ApJ, 841, 125

Joshi, B. C., Arumugasamy, P., Bagchi, M., et al. 2018, JApA, 39, 51

Kerr, M., Reardon, D. J., Hobbs, G., et al. 2020, PASA, 37, e020

Kirsten, F., Bhat, N. D. R., Meyers, B. W., et al. 2019, ApJ, 874, 179

Krishnakumar, M. A., Manoharan, P. K., Joshi, B. C., et al. 2021, arXiv:2101. 05334

Kumar, U., Gupta, Y., van Straten, W., et al. 2013, in IAU Symp. 291: Neutron Stars and Pulsars: Challenges and Opportunities after 80 years, ed. J. van Leeuwen (New York: IAU), 432

Lam, M. T. 2017, Pulse: PSRFITS handler, Astrophysics Source Code Library ascl: 1706.011

Lam, M. T., Cordes, J. M., Chatterjee, S., et al. 2016, ApJ, 821, 66

Lam, M. T., McLaughlin, M. A., Cordes, J. M., Chatterjee, S., \& Lazio, T. J. W. 2018, ApJ, 861, 12

Lambert, H. C., \& Rickett, B. J. 1999, ApJ, 517, 299

Lasky, P. D., Mingarelli, C. M. F., Smith, T. L., et al. 2016, PhRvX, 6, 011035

Levin, L., McLaughlin, M. A., Jones, G., et al. 2016, ApJ, 818, 166

Lorimer, D. R., \& Kramer, M. 2012, Handbook of Pulsar Astronomy (Cambridge: Cambridge Univ. Press)

Manchester, R. N., Hobbs, G. B., Teoh, A., \& Hobbs, M. 2005, AJ, 129, 1993

Nice, D., Demorest, P., Stairs, I., et al. 2015, Tempo: Pulsar timing data analysis, Astrophysics Source Code Library, ascl:1509.002

Perera, B. B. P., DeCesar, M. E., Demorest, P. B., et al. 2019, MNRAS, 490, 4666

Roy, J., Gupta, Y., Pen, U.-L., et al. 2010, ExA, 28, 25

Sesana, A. 2013, MNRAS, 433, L1

Turner, J. E., McLaughlin, M. A., Cordes, J. M., et al. 2020, arXiv:2012.09884 van Straten, W. 2006, ApJ, 642, 1004

van Straten, W., \& Bailes, M. 2010, DSPSR: Digital Signal Processing Software for Pulsar Astronomy, Astrophysics Source Code Library, ascl:1010.006

van Straten, W., Demorest, P., Khoo, J., et al. 2011, PSRCHIVE: Development Library for the Analysis of Pulsar Astronomical Data, Astrophysics Source Code Library, ascl:1105.014

van Straten, W., Demorest, P., \& Oslowski, S. 2012, AR\&T, 9, 237

Yao, J. M., Manchester, R. N., \& Wang, N. 2017, ApJ, 835, 29 